\title{
Potential Application of MicroRNA Profiling to the Diagnosis and Prognosis of HIV-1 Infection
}

\author{
Bin Su ${ }^{1,2+}$, Yuping $\mathrm{Fu}^{3+}$, Yan Liu' ${ }^{1,2}$, Haoquan $\mathrm{Wu}^{4}$, Ping Ma ${ }^{5}$, Weiping Zeng ${ }^{6}$, \\ Tong Zhang ${ }^{1,2 *}$, Shi Lian ${ }^{3 *}$ and Hao $\mathrm{Wu}^{1,2 *}$ \\ ${ }^{1}$ Center for Infectious Diseases, Beijing Youan Hospital, Capital Medical University, Beijing, China, ${ }^{2}$ Beijing Key Laboratory \\ for HIVIAIDS Research, Beijing, China, ${ }^{3}$ Department of Dermatology, Xuanwu Hospital, Capital Medical University, Beijing, \\ China, ${ }^{4}$ Kanglin Biotech (Hangzhou) Co., Ltd., Zhejiang, China, ${ }^{5}$ Department of Infectious Diseases and STDs, Tianjin \\ Second People's Hospital, Tianjin, China, ${ }^{6}$ Department of Biochemistry and Microbiology, Marshall University School \\ of Medicine, Huntington, WV, United States
}

\section{OPEN ACCESS}

Edited by:

Petros Karakousis,

Johns Hopkins University,

United States

Reviewed by:

Hector Ariel Roldan,

McGill University, Canada

Charani Ranasinhe

Australian National University,

Australia

*Correspondence:

Tong Zhang

zt_doc@ccmu.edu.cn

Shi Lian

drlianshi@sina.com

Hao Wu

whdoc@ccmu.edu.cn

tThese authors have contributed equally to this work

Specialty section:

This article was submitted to Infectious Diseases,

a section of the journal

Frontiers in Microbiology

Received: 04 September 2018 Accepted: 10 December 2018

Published: 21 December 2018

Citation:

Su B, Fu Y, Liu Y, Wu H, Ma P, Zeng W, Zhang T, Lian S and Wu H

(2018) Potential Application of MicroRNA Profiling to the Diagnosis and Prognosis of HIV-1 Infection.

Front. Microbiol. 9:3185.

doi: 10.3389/fmicb.2018.03185
MicroRNAs (miRNAs) were first identified in Caenorhabditis briggsae and later recognized as playing pivotal roles in a vast range of cellular activities. It has been shown that miRNAs are an important mechanism not only for host defense against virus but also for the establishment of viral infection. During human immunodeficiency virus type 1 (HIV-1) infection, host miRNA profiles are altered either as a host response against the virus or alternatively as a mechanism for the virus to facilitate viral replication and infection or to maintain latency. The altered miRNA profiles can be detected and quantified by various advanced assays, and potentially serve as more sensitive, accurate and cost-efficient biomarkers for HIV-1 diagnosis and disease progression than those detected by currently available standard clinical assays. Such new biomarkers are critical for optimizing treatment regimens. In this review, we focus on the potential application of miRNA profiling to the diagnosis of HIV-1 infection and the monitoring of disease progression.

Keywords: HIV-1, microRNA, disease progression, biomarker, immune system

\section{INTRODUCTION}

MicroRNAs (miRNAs) are small non-coding RNAs that regulate gene expression. In the human genome, more than 1500 different miRNAs have been identified, which collectively regulate the expression of about one third of all human genes (Li and Zhang, 2013). miRNAs regulate diverse biological functions, such as cellular differentiation, development, apoptosis, tumorigenesis, and targeting of foreign pathogens (Ouellet et al., 2009; Berkhout and Liu, 2014). miRNA research has advanced considerably in recent years. It has been shown that the expression of many miRNAs is altered during human immunodeficiency virus type 1 (HIV-1) infection. Changes in miRNA expression in infected humans can be detected with various advanced assays (Affymetrix ${ }^{\mathrm{TM}}$ assay, digital droplet $\mathrm{qPCR}$, etc., and sequencing). Therefore, miRNAs can potentially be used to detect HIV-1 infection and monitor disease progression with greater sensitivity and efficiency than conventional methods. This review provides an overview of current knowledge concerning miRNA and its relation to HIV-1 infection and disease progression. A model of the miRNA biogenesis pathway and its correlation with $\mathrm{CD} 4^{+}$ 
T-cell count, T-cell receptor expression, serum viral load, and virus replication is proposed in Figure 1.

\section{THE MIRNA BIOGENESIS PATHWAY}

The genes encoding miRNAs are dispersed throughout the human genome (Rodriguez et al., 2004). Many miRNAs are generated as the sole transcripts of non-coding regions. Genes encoding miRNAs may also occur within introns or untranslated regions of protein-coding DNA (Poliseno et al., 2010; Ramalingam et al., 2014). The genes transcribed by RNA polymerase II can produce messenger RNAs (mRNAs) or regulatory RNAs called primary RNAs (Lee et al., 2004), which form a typical hairpin loop structure and undergo several processing steps to become miRNAs (Quick-Cleveland et al., 2014). The processing begins with recognition of the doublestranded stem by the DiGeorge syndrome chromosomal region 8 (DGCR8) protein. An enzyme called DROSHA then associates with DGCR8 to form a complex that cleaves the RNA to generate a smaller precursor miRNA (Yang and Lai, 2011; Wu et al., 2012). The precursor miRNA is transported from the nucleus to the cytoplasm by the transporter protein exportin 5 and is then immediately recognized by an RNase called Dicer. Dicer cleaves the hairpin loop structure to form a short double-strand miRNA molecule. The Argonaut protein then interacts with Dicer and binds the miRNA. The miRNA is unwound and one strand, the "star strand" or "passenger strand," is released and degraded. The remaining strand, the "guide strand," which is typically 19-22 nucleotides long, interacts with several proteins to form the RNA-induced silencing complex (RISC). The guide strand guides the RISC complex to its target sequences in mRNA, enabling it to inactivate one or several genes. The mRNA of the target gene is complementary to the essential "seed" region of the miRNA, which is often $2 \sim 8$ nucleotides long, facilitating partial complementary base pairing with the mRNA. Once bound, the RISC can block mRNA translation by preventing the ribosome subunit from binding to the mRNA. As a result, the mRNA is not translated into protein and the gene is effectively silenced (Swaminathan et al., 2012a; Fukaya et al., 2014; Zinovyeva et al., 2015). The vital role of miRNA in a vast range of biological processes opens up tremendous possibilities for new treatments and diagnosis of various diseases in humans. We focus here on the potential of miRNA for the diagnosis of HIV-1 infection and monitoring of its progression.

\section{THE ROLE OF miRNAs IN HIV-1 INFECTION}

HIV-1 mainly infects human $\mathrm{CD}^{+} \mathrm{T}$ cells through the recognition of the $\mathrm{CD} 4^{+}$receptor on the cell surface. The virus gradually reduces the number of $\mathrm{CD} 4^{+} \mathrm{T}$ cells in the infected patient, which impairs the human immune system and eventually leads to acquired immunodeficiency syndrome (AIDS) (Weiss, 1993; Douek et al., 2009). After gaining entry to the body via the intravenous route or lesions in mucosal surfaces, HIV-1 binds to the CD4 receptor and the CCR5 or CXCR4 co-receptors on immune cells. The virus then penetrates the cell membrane and releases its RNA genome into the cell, which is subsequently reverse-transcribed into DNA and integrated into the host cell genome (Haase, 2011). Viral particles are produced in the infected cells, and disseminate from the initial site of infection to establish systemic infection. At late stages of infection, which are characterized by high viral load and low $\mathrm{CD}^{+}$ T-cell counts in the blood, the patient becomes susceptible to infection with secondary pathogens, such as Pneumocystis carinii, Candida albicans, and Mycobacterium tuberculosis, eventually succumbing to AIDS (Aaron et al., 2004). HIV-1 replication within host cells is regulated by various host factors, including miRNAs, which may target virus mRNAs directly or regulate the expression of host proteins that HIV-1 hijacks for its own replication. The miRNA profile of the host changes during infection, with different miRNAs produced at different stages of disease progression in HIV-1-infected individuals (Swaminathan et al., 2012a).

\section{THE EFFECT OF HIV INFECTION ON MIRNA EXPRESSION}

HIV-1 infection is known to trigger changes in the expression of many miRNAs, either by influencing host cellular activities or as a result of the cellular response to HIV-1 infection (Balasubramaniam et al., 2018). The ability of miRNA analyses to distinguish between individuals with and without HIV-1 infection has been reported worldwide. In 2012, Gupta et al. (2011) analyzed 704 kinds of miRNA in peripheral blood mononuclear cells (PBMCs) of HIV-1-infected individuals and healthy controls. They found that 28 of these miRNAs were up-regulated and 14 were down-regulated in HIV-infected individuals. Using Target Scan software, they predicted that the targets of these miRNAs were mostly related to the cell cycle, proliferation, movement, migration, and signal transduction. The altered transcriptional profiles of these miRNAs may lead to various non-AIDS disorders (Gupta et al., 2011). On the other hand, HIV-1 gp120 and tat were recently shown to accelerate endothelial cell senescence by disrupting senescence-associated miRNAs, thereby increasing the risk of atherosclerotic vascular disease. This discovery provides further support for the notion that, as $\mathrm{CD} 4^{+} \mathrm{T}$ cells are the main targets of HIV- 1 infection, they should contain miRNAs that are differentially expressed in the presence and absence of HIV-1 infection. Swaminathan's team demonstrated significant down-regulation of the Let-7 family of miRNAs in patients with chronic HIV-1 infection (Swaminathan et al., 2012b). In the same year, a study on elite controllers led by Witwer et al. (2012) showed that miR-125b and -150 levels were strongly correlated with HIV-1 viral load in HIV-1infected patients. Amaral et al. (2017) recently discovered that levels of miR-34c-5p, a miRNA responding to T-cell receptor stimulation in naïve $\mathrm{T}$ cells, were decreased during the disruption of host immune responses by HIV-1. In 2014, researchers analyzed subcutaneous adipose tissue from eight HIV-1-infected 


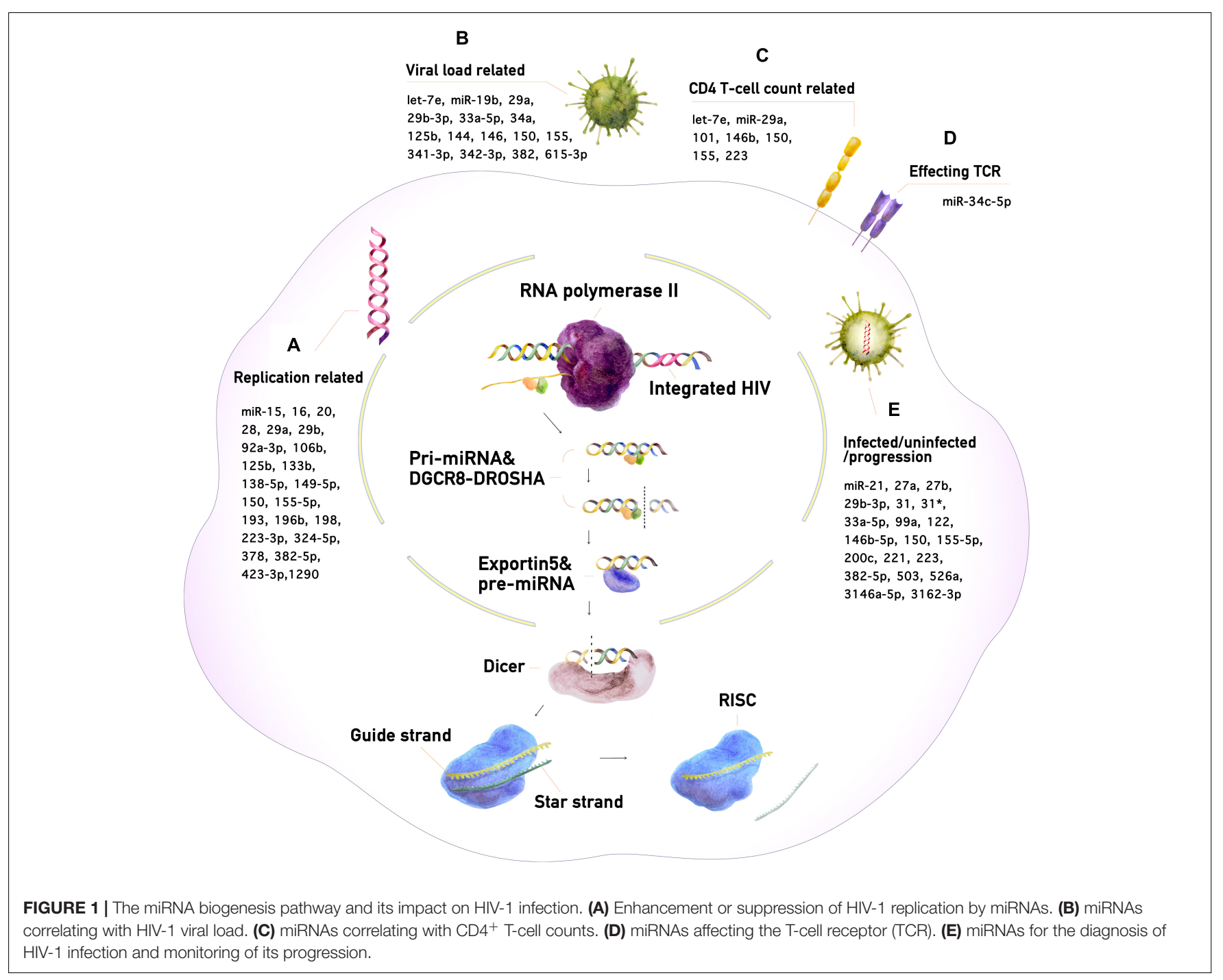

patients. They used human miRNA microarrays to probe the transcriptomes of the samples and found that 21 of 754 miRNAs were overexpressed, with 10 of these miRNAs displaying at least 2.5-fold up-regulation (Squillace et al., 2014). Thapa et al. (2014) suggested that serum miR-21, miR-122, and miR-223 levels can be used to distinguish between HIV-1-infected and uninfected individuals, and that miR-222 can also be used as a diagnostic marker for AIDS-associated non-Hodgkin lymphoma.

\section{THE ROLE OF miRNAs IN HIV-1 REPLICATION}

MicroRNAs can affect $\mathrm{HIV}-1$ infection through multiple mechanisms. Anti-HIV-1 miRNAs repress HIV-1 activities through CCR5 or CXCR4, the auxiliary receptors for HIV1, or target HIV-1 directly via the env, pol, gag, vif, and tat genes of the HIV-1 genome. Hariharan et al. (2005) used a consensus scoring method and showed that miR-29a and miR29b targeted nef, miR-149 targeted $v p r$, miR-378 targeted $e n v$, and
miR-324-5p targeted vif within the HIV-1 genome. Huang et al. (2007) discovered that miR-28, miR-125b, miR-150, miR-223, and miR-382-5p targeted the $3^{\prime}$ UTR region of the HIV-1 mRNA in cells, which decreases $\mathrm{CD}^{+}{ }^{+} \mathrm{T}$-cell activation during the resting period (Huang et al., 2007). Monocytes are rich in these anti-HIV-1 miRNAs, which are down-regulated in macrophages after differentiation, rendering macrophages susceptible to HIV1 infection. The suppression of these miRNAs increases the susceptibility of mononuclear cells to HIV-1 infection (Hariharan et al., 2005; Huang et al., 2007). The $3^{\prime}$ UTR region of the HIV1 RNA genome has been identified as the target of miR-196b and miR-1290 (Wang et al., 2015). The inhibition of these two miRNAs can lead to the activation of latent HIV-1, leading to viral clearance of latent viral reservoirs by virus-induced cytolysis and host antiviral immune responses in the presence of antiretroviral therapy (ART) (Mbonye and Karn, 2014; Wang et al., 2015, 2018).

Some miRNAs can also enhance HIV-1 infection. Chiang et al. (2013) found that miR-132 was significantly more strongly expressed in activated $\mathrm{CD} 4^{+} \mathrm{T}$ cells than in steady-state $\mathrm{CD} 4^{+}$ 
T cells. Although the underlying mechanism for this remains unknown, in this study they found that miR-132 enhanced HIV1 replication and increased the susceptibility of activated $\mathrm{CD} 4{ }^{+}$ T cells to HIV-1 infection (Chiang et al., 2013). miR-217 and miR-34a can significantly increase tat levels, and can also bind to mRNA of the SIRT1 gene (Kwon and Ott, 2008) and inhibit its expression, thereby enhancing the transcriptional activation mediated by HIV-1 tat (Zhang et al., 2012).

MicroRNAs also indirectly regulate the effects of HIV-1 infection by targeting the HIV-dependent factors interacting with HIV-1. The histone acetyltransferase P300 and P300-CREB binding protein-associated factor (PCAF) are required for tat acetylation, leading to the up-regulation of the transcription of the HIV-1 LTR. Cell-associated miR-17/92 targets PCAF and inhibits viral replication (Triboulet et al., 2007). miR198 plays an anti-HIV-1 role by down regulating cyclin T1, an important component of the eukaryotic RNA polymerase II prolongation complex. This protein forms a heterodimer with CDK9 (transcription elongation factor B or $\mathrm{p}$-TEFb). The p-TEFb complexes interact with HIV-1 transactivating response (TAR) elements and tat, promoting viral transcription. Cyclin $\mathrm{T} 1$ is more strongly expressed in HIV-1-susceptible cells, such as macrophages, than in mononuclear cells (Sung and Rice, 2009). Pur-alpha is a sequence-specific DNA and RNA binding protein that binds to the HIV-1 TAR element and tat in HIV1 -infected nuclei, up regulating viral transcription (Chepenik et al., 1998). Unlike macrophages and dendritic cells, which differentiate from monocytes, monocytes themselves displayed a significant repression of Pur-alpha. This repression is due to the inhibitory effect of certain miRNAs such as miR-15a, miR-15b, miR-16, miR-20a, miR-193, and miR-106b targeting Pur-alpha, and consequentially decreased the susceptibility to HIV-1 infection of monocytes compared to their macrophage "descendants" (Shen et al., 2012).

More interestingly, some studies have reported that the interference capacity of miRNAs is not restricted to gene silencing. Some miRNAs can bind to the nucleocapsid protein. Research led by Chen discovered that certain miRNAs interfere with HIV-1 replication by binding to the gag protein and intercepting HIV-1 assembly, rather than by forming RISCs and recognizing mRNAs (Chen et al., 2014).

Most of the mentioned miRNAs function by suppressing HIV1 replication, but others may promote this process. Either way, the shift in miRNA profile caused by HIV-1 infection or as part of the host immune response indicates a change in the capability of HIV-1 to replicate that is closely related to the viral load.

\section{HIV-1-ENCODED miRNAs}

Viral genomes may also encode their own miRNAs, which could be even more useful than human miRNAs for the diagnosis of viral infection. The first viral miRNAs to be described, in 2004, were from Epstein-Barr virus (Pfeffer et al., 2004). However, given that HIV-1 is an RNA virus, it remains unclear whether it encodes its own miRNAs. A well accepted view is that the RNA-based HIV-1 genome might be inactivated by its own miRNAs or the intermediate products during the processing of these miRNAs, thereby interfering with the replication of these miRNA-encoding sequences (Umbach et al., 2010; Whisnant et al., 2013; Harwig et al., 2014). Nevertheless, evidence for HIV-1-encoded miRNAs has been accumulating over the last few years. These viral miRNAs may play a role in the versatility of the viruses and their ability to hijack host cellular pathways for their own purposes.

The first candidates of HIV-1-encoded miRNAs were identified in an in silico analysis by Bennasser et al. (2004) only a few months after the description of the first viral miRNA. This analysis of the HIV-1 genome predicted a few possible viral miRNAs encoded by HIV-1 (Bennasser et al., 2004). Couturier and Root-Bernstein (2005) subsequently suggested that HIV may produce miRNAs that inhibit the expression of some cell surface markers of clusters of differentiation (CD) and interleukins (IL). More recently, HIV-1 has been shown to produce a replicationenhancing miRNA from its reverse transcriptase region (Zhang et al., 2014). The debate about whether or not HIV-1 can produce its own miRNA thus continues, with evidence on both sides, but these potential viral miRNAs merit further investigation.

\section{CHANGES IN miRNA LEVELS IN PBMCS AND SERUM FROM PATIENTS BASED ON HIV DISEASE PROGRESSION}

In the studies led by Houzet, the patients were assigned to four groups on the basis of $\mathrm{CD}^{+}$T-cell counts, viral load, and the miRNA expression profiles of PBMCs in HIV-1-infected individuals. In total, 63 different miRNAs displayed changes in levels in the HIV-1-infected PBMCs, indicating differential expression of specific miRNAs among the different groups. For example, let-7e, miR-101, miR-146b, and miR-155 were found only in the low $\mathrm{CD} 4^{+}$T-cell count and high-viral load group. The levels of miR-223, miR-150, miR-146b, miR-16, and miR191 in $\mathrm{T}$ cells were lower, by a factor of three to nine, in the PBMCs of patients with different disease progression profiles. This observation suggested that specific miRNAs are potential biomarkers for differentiating the stages of progression of HIV1 infection (Houzet et al., 2008). Dey et al. (2016) compared the expression of miRNAs in PBMCs between long-term nonprogressors (LTNP), slow progressors, and rapid progressors. Levels of miR-382-5p were lower in LTNP and displayed a positive linear correlation with plasma viral load. A combination of miR-382-5p and miR-155-5p can be used to distinguish between LTNP and chronic progressors, and between infected and non-infected individuals. Decreases in the levels of miR382-5p and miR-155-5p are beneficial for the control of disease progression (Dey et al., 2016).

About $10 \sim 15 \%$ of HIV-1-infected patients display a rapid decrease in $\mathrm{CD}^{+}$T-cell count at early stages of infection, with rapid progression to AIDS. A comparison of the miRNA expression profiles in the PBMCs of rapid progressors and slow progressors revealed differences in miR-31, miR-200c, miR-526a, miR-99a, and miR-503 levels that could be used as biomarkers for rapid progressors, favoring early intervention (Sung and Rice, 2009). Duskova et al. (2013) found that miR-19b, miR-146a, 
miR-615-3p, miR-382, miR-34a, miR-144, and miR-155, which regulate innate immunity- and inflammation-related genes, were significantly up-regulated in PBMCs with a high viral load in HIV-1-infected patients.

Munshi et al. (2014) compared the levels of expression of miR-16, miR-146b-5p, miR-150, miR-191, miR-223, and miR$146 \mathrm{~b}-5 \mathrm{p}$ in plasma between patients without AIDS, patients with AIDS who had not received ART, patients who had received ART and patients who had received ART but displayed drug resistance. They found that miR-146b-5p levels in PBMCs and plasma not only distinguished different progression groups, but also predicted reactivity to ART. In addition, miR-150 could be used as an index for monitoring the progression of HIV/AIDS disease and the effect of ART (Munshi et al., 2014). Rosca et al. (2016) found that levels of miR-29a were inversely correlated with HIV viral load and the degree of immunosuppression (the number of $\mathrm{CD}^{+} \mathrm{T}$ cells and $\mathrm{CD}^{+}{ }^{+} \mathrm{T} / \mathrm{CD} 8^{+} \mathrm{T}$ ratio) in 165 young subjects with chronic HIV-1 infection. Levels of miR-29a and responses to treatment are different among patients. Levels of miR-29a were low in patients who had experienced treatment failure (CD4 $<350$ cells/ $\mu \mathrm{l})$. miR-29a thus can be used as a biological marker of long-term survivors and patients who have received ART, and it can also be used to predict disease prognosis and progression (Rosca et al., 2016).

The small proportion of elite controllers among HIV-infected patients can effectively control the infection without drug treatment. Elite controllers are infected with HIV but able to sustain undetectable viral loads and usually normal CD4 ${ }^{+} \mathrm{T}$-cell count without any treatment (Walker, 2007; Autran et al., 2011). Witwer et al. (2012) found that a difference in miR-31 and miR$31^{*}$ levels between the PBMCs of patients with HIV-1 viremia and elite controllers reflected the progression of HIV-1 infection. miR-31 levels were positively correlated with $\mathrm{CD} 4^{+} \mathrm{T}$-cell count, whereas miR-341-3p levels were negatively correlated with viral load. This result suggests the levels of these miRNAs are potential biomarkers of the risk of progression of HIV-1 infection (Witwer et al., 2012). Reynoso et al. (2014) compared the changes in plasma levels of miRNAs in elite controllers, individuals with chronic HIV-1 infection and healthy individuals. They found that plasma miR-29b-3p, miR-33a-5p, and miR-3146a-5p levels in elite controllers were significantly higher than those in patients with chronic infection. Further experiments in vitro showed that the overexpression of miR-29b-3p and miR-33a-5p could reduce HIV-1 production. Egana-Gorrono et al. (2014) analyzed the expression profiles of 286 different human miRNAs in elite controllers, individuals with viremia, individuals who received ART and healthy individuals: 23 different miRNAs displayed differential expression between the elite controller and viremia groups. These miRNAs included miR-221, miR-27a, miR-27b, and miR-29b, which were up-regulated in the serum of elite controllers and HIV-1-negative individuals, but were downregulated in the serum of patients with viremia and HIV1 -infected patients on ART. Moreover, another 19 different miRNAs were down-regulated in the serum of elite controllers and HIV-1-negative individuals, and up-regulated in the serum of individuals with viremia and HIV-1-infected individuals on ART (Egana-Gorrono et al., 2014).
The change of miRNA profiles in $\mathrm{CD}^{+} \mathrm{T}$ cells is even more closely correlated with disease progression since the $\mathrm{CD} 4^{+}$ $\mathrm{T}$ cells are the main targets of HIV-1 infection. Interferoninducible protein 10 (IP-10) is a crucial inflammatory cytokine that can trigger immune dysfunction and disease progression during HIV-1 infection. By comparing the sequences of the gene encoding IP-10 and those of human miRNAs, Wu et al. (2017) recently found that six miRNAs matched the sequence of the IP-10 $3^{\prime}$ UTR. Further analysis revealed that one of these miRNAs, miR-21, was down-regulated in monocytes during HIV1 infection and responsible for the increase in IP-10 levels leading to inflammation and the rapid loss of $\mathrm{CD} 4^{+} \mathrm{T}$ cells, which is closely related to disease progression (Wu et al., 2017).

\section{THE RELATIONSHIP BETWEEN miRNAS AND DISEASE PROGRESSION IN HIV-1-INFECTED INDIVIDUALS}

MicroRNA has been shown to correlate with viral latency and disease progression. Many researchers found distinct miRNA profile during different stages of disease progression, and levels of certain miRNAs may influence the susceptibilities of certain cells (as discussed in the section regarding PBMCs and serum) as well as the speed of disease progression period.

Host miRNA is very important for HIV-1 latency. For example, the $\mathrm{CD}^{+}{ }^{+} \mathrm{T}$ cells of HIV-1-infected individuals in the LTNPs and untreated patients groups display changes in miR-155 level (Bignami et al., 2012). The Let-7 family of miRNAs targets IL-10, a pleiotropic cytokine. Let-7 is down-regulated in chronic progressors (Zhang et al., 2016). IL-2 is also a major factor for the maintenance of the activation, survival and replication of $\mathrm{CD} 4^{+}$ $\mathrm{T}$ cells. The levels of miR-9 in CD4 ${ }^{+} \mathrm{T}$ cells from individuals with chronic HIV-1 infection are lower than those in healthy individuals or LTNPs. This decrease in miR-9 levels triggers an increase in mature protein 1 in $\mathrm{B}$ cells, leading to the inhibition of IL-2 production and promoting disease progression, which is also evidence for the positive role of miR-9 in maintaining HIV-1 latency (Seddiki et al., 2013). These findings suggest that longterm monitoring of miR-155 might be very valuable for assessing HIV-1 latency status in infected individuals.

Many other factors are correlated with immune status and disease progression. The abundance and size of extracellular vesicles are correlated with the apoptosis and pyroptosis of $\mathrm{CD}_{4}^{+}$ T cells and with miR-155 and miR-223 levels, as shown by Hubert et al. (2015). As the authors suggested, analysis of these miRNAs, together with plasma exosome abundance, extracellular vesicles and size may provide accurate fingerprint of disease progression (Hubert et al., 2015).

The expression of miRNAs in cells and body fluids has been shown to be associated with the severity and progression of HIV infection. On the basis of $\mathrm{CD}_{4}{ }^{+}$T-cell counts, viral load and whether ART has been administered, HIV-1-infected patients can be divided into typical progressors, rapid progressors, slow progressors, LTNP and HIV-infected individuals in the early stages of antiretroviral treatment (Li et al., 2010). Some miRNAs are differentially expressed in these different groups, and their 
abnormal expression can serve as a biomarker of the disease progression and provide important information about prognosis (Dey et al., 2016).

For instance, Munshi's team described miR-150 as a potential biomarker for HIV/AIDS disease progression and treatment monitoring. They compared its levels in PBMCs and plasma between healthy donors, asymptomatic patients, symptomatic patients, patients on ART and patients who had developed drug resistance. They found that this particular miRNA was differentially expressed among these groups (Munshi et al., 2014). Similarly, in a very recent study, Huang et al. (2018) collected plasma from healthy donors, patients infected for less than 1 year and patients infected for more than 1 year. Differential expression of miR-3162-3p was observed between these groups. The clinical significance of this discovery was validated by successfully predicting disease progression based on the expression of the miRNA and in an in vitro model (Huang et al., 2018).

\section{CONCLUDING REMARKS AND REMAINING QUESTIONS}

This review highlights the value of miRNA for the diagnosis of HIV-1 infection and the evaluation of disease progression. We have considered the effects of miRNA on HIV-1 replication and of HIV-1 infection on miRNA profiles in different cell subsets. In each case, there is growing evidence for differences in miRNA levels directly or indirectly due to HIV-1 infection. While standard testing methods have their limitations such as in detecting low viral load, monitoring miRNA may provide valuable information for diagnosis and predicting HIV/AIDS progression. It may now be feasible to establish a national or international database or guideline for miRNA-based disease diagnosis. However, there are still many obstacles. Some of the studies mentioned in this article suggest that miRNA profiles are not entirely homogeneous within a given group and that many miRNA levels are individual-dependent, with changes in

\section{REFERENCES}

Aaron, L., Saadoun, D., Calatroni, I., Launay, O., Memain, N., Vincent, V., et al. (2004). Tuberculosis in HIV-infected patients: a comprehensive review. Clin. Microbiol. Infect. 10, 388-398. doi: 10.1111/j.1469-0691.2004.00758.x

Amaral, A. J., Andrade, J., Foxall, R. B., Matoso, P., Matos, A. M., Soares, R. S., et al. (2017). miRNA profiling of human naive CD4 T cells links miR-34c-5p to cell activation and HIV replication. EMBO J. 36, 346-360. doi: 10.15252/embj. 201694335

Autran, B., Descours, B., Avettand-Fenoel, V., and Rouzioux, C. (2011). Elite controllers as a model of functional cure. Curr. Opin. HIV AIDS 6, 181-187. doi: 10.1097/COH.0b013e328345a328

Balasubramaniam, M., Pandhare, J., and Dash, C. (2018). Are microRNAs important players in HIV-1 infection? An update. Viruses 10:E110. doi: 10.3390/ v10030110

Bennasser, Y., Le, S. Y., Yeung, M. L., and Jeang, K. T. (2004). HIV-1 encoded candidate micro-RNAs and their cellular targets. Retrovirology 1:43. doi: 10. 1186/1742-4690-1-43

Berkhout, B., and Liu, Y. P. (2014). Towards improved shRNA and miRNA reagents as inhibitors of HIV-1 replication. Fut. Microbiol. 9, 561-571. doi: $10.2217 / \mathrm{fmb} .14 .5$ their profiles not directly due to HIV-1 infection. In addition, some of the studies described here were performed ex vivo, and confirmation of their results is required in vivo and in clinical trials. Furthermore, according to some reports, HIV-1 may also be able to develop resistance to miRNA interference (Boden et al., 2003; Westerhout et al., 2005), which would complicate the pursuit of approaches based on the use of miRNA biomarkers. With the development of highly sensitive assays and high-throughput sequencing, advances are being made in the field of miRNAs. While there are still some pieces missing from the puzzle before miRNAs can be used as clinical biomarkers for HIV-1 infection and HIV/AIDS disease progression, many studies, as reviewed in this article, have demonstrated the potentials of miRNAs as biomarkers for these clinical processes.

\section{AUTHOR CONTRIBUTIONS}

All authors listed have made a substantial, direct, and intellectual contribution to the work and read and approved the final manuscript.

\section{FUNDING}

This work was supported by the National 13th FiveYear Grand Program on Key Infectious Disease Control (2017ZX10202102-005-003 to BS, 2017ZX10202101-004-001 to TZ, 2017ZX10202102-005-001 to HaW, and 2017ZX10202102005-004 to PM), the National Natural Science Foundation of China (NSFC, 81772165 to BS and 81571973 to HW), the Funding for Chinese overseas talents returning to China in 2016 (to BS), the Basic-Clinical Research Cooperation Fund of Capital Medical University (17JL20 to BS), and the Beijing Key Laboratory for HIV/AIDS Research (BZ0089). The funders had no role in study design, data collection and analysis, decision to publish, or preparation of the manuscript.

Bignami, F., Pilotti, E., Bertoncelli, L., Ronzi, P., Gulli, M., Marmiroli, N., et al. (2012). S\changes in CD4+ T lymphocyte miRNA expression after exposure to HIV-1. Blood 119, 6259-6267. doi: 10.1182/blood-2011-09-379503

Boden, D., Pusch, O., Lee, F., Tucker, L., and Ramratnam, B. (2003). Human immunodeficiency virus type 1 escape from RNA interference. J. Virol. 77, 11531-11535. doi: 10.1128/JVI.77.21.11531-11535.2003

Chen, A. K., Sengupta, P., Waki, K., Van Engelenburg, S. B., Ochiya, T., Ablan, S. D., et al. (2014). MicroRNA binding to the HIV-1 gag protein inhibits gag assembly and virus production. Proc. Natl. Acad. Sci. U.S.A. 111, E2676-E2683. doi: 10.1073/pnas.140803 7111

Chepenik, L. G., Tretiakova, A. P., Krachmarov, C. P., Johnson, E. M., and Khalili, K. (1998). The single-stranded DNA binding protein, Pur-alpha, binds HIV-1 TAR RNA and activates HIV1 transcription. Gene 210, 37-44. doi: 10.1016/S0378-1119(98)00 033-X

Chiang, K., Liu, H., and Rice, A. P. (2013). miR-132 enhances HIV-1 replication. Virology 438, 1-4. doi: 10.1016/j.virol.2012.12.016

Couturier, J. P., and Root-Bernstein, R. S. (2005). HIV may produce inhibitory microRNAs (miRNAs) that block production of CD28, CD4 and some interleukins. J. Theor. Biol. 235, 169-184. doi: 10.1016/j.jtbi.2005.01.001 
Dey, R., Soni, K., Saravanan, S., Balakrishnan, P., Kumar, V., Boobalan, J., et al. (2016). Anti-HIV microRNA expression in a novel Indian cohort. Sci. Rep. 6:28279. doi: $10.1038 /$ srep28279

Douek, D. C., Roederer, M., and Koup, R. A. (2009). Emerging concepts in the immunopathogenesis of AIDS. Annu. Rev. Med. 60, 471-484. doi: 10.1146/ annurev.med.60.041807.123549

Duskova, K., Nagilla, P., Le, H. S., Iyer, P., Thalamuthu, A., Martinson, J., et al. (2013). MicroRNA regulation and its effects on cellular transcriptome in human immunodeficiency virus-1 (HIV-1) infected individuals with distinct viral load and CD4 cell counts. BMC Infect. Dis. 13:250. doi: 10.1186/1471-2334-13-250

Egana-Gorrono, L., Escriba, T., Boulanger, N., Guardo, A. C., Leon, A., Bargallo, M. E., et al. (2014). Differential microRNA expression profile between stimulated PBMCs from HIV-1 infected elite controllers and viremic progressors. PLoS One 9:e106360. doi: 10.1371/journal.pone.0106360

Fukaya, T., Iwakawa, H. O., and Tomari, Y. (2014). MicroRNAs block assembly of eIF4F translation initiation complex in Drosophila. Mol. Cell. 56, 67-78. doi: 10.1016/j.molcel.2014.09.004

Gupta, A., Nagilla, P., Le, H. S., Bunney, C., Zych, C., Thalamuthu, A., et al. (2011). Comparative expression profile of miRNA and mRNA in primary peripheral blood mononuclear cells infected with human immunodeficiency virus (HIV-1). PLoS One 6:e22730. doi: 10.1371/journal.pone.0022730

Haase, A. T. (2011). Early events in sexual transmission of HIV and SIV and opportunities for interventions. Annu. Rev. Med. 62, 127-139. doi: 10.1146/ annurev-med-080709-124959

Hariharan, M., Scaria, V., Pillai, B., and Brahmachari, S. K. (2005). Targets for human encoded microRNAs in HIV genes. Biochem. Biophys. Res. Commun. 337, 1214-1218. doi: 10.1016/j.bbrc.2005.09.183

Harwig, A., Das, A. T., and Berkhout, B. (2014). Retroviral microRNAs. Curr. Opin. Virol. 7, 47-54. doi: 10.1016/j.coviro.2014.03.013

Houzet, L., Yeung, M. L., de Lame, V., Desai, D., Smith, S. M., and Jeang, K. T. (2008). MicroRNA profile changes in human immunodeficiency virus type 1 (HIV-1) seropositive individuals. Retrovirology 5:118. doi: 10.1186/1742-46905- 118

Huang, J., Lai, J., Liang, B., Jiang, J., Ning, C., Liao, Y., et al. (2018). mircoRNA$3162-3 p$ is a potential biomarker to identify new infections in HIV-1-infected patients. Gene 662, 21-27. doi: 10.1016/j.gene.2018.04.002

Huang, J., Wang, F., Argyris, E., Chen, K., Liang, Z., Tian, H., et al. (2007). Cellular microRNAs contribute to HIV-1 latency in resting primary CD4+ T lymphocytes. Nat. Med. 13, 1241-1247. doi: 10.1038/nm1639

Hubert, A., Subra, C., Jenabian, M. A., Tremblay Labrecque, P. F., Tremblay, C., Laffont, B., et al. (2015). Elevated abundance, size, and MicroRNA content of plasma extracellular vesicles in viremic HIV-1+ patients: correlations with known markers of disease progression. J. Acquir. Immune Defic. Syndr. 70, 219-227. doi: 10.1097/QAI.0000000000000756

Kwon, H. S., and Ott, M. (2008). The ups and downs of SIRT1. Trends Biochem. Sci. 33, 517-525. doi: 10.1016/j.tibs.2008.08.001

Lee, Y., Kim, M., Han, J., Yeom, K. H., Lee, S., Baek, S. H., et al. (2004). MicroRNA genes are transcribed by RNA polymerase II. EMBO J. 23, 4051-4060. doi: 10.1038/sj.emboj.7600385

Li, D. M., Wang, L., Gao, X., Wang, Z., Cui, Z. L., Song, L. P., et al. (2010). [Study on the natural history of HIV among former commercial plasma donors caused by contaminated plasma donation in central China]. Zhonghua Liu Xing Bing Xue Za Zhi 31, 633-637.

Li, J., and Zhang, Z. (2013). miRNA regulatory variation in human evolution. Trends Genet. 29, 116-124. doi: 10.1016/j.tig.2012.10.008

Mbonye, U., and Karn, J. (2014). Transcriptional control of HIV latency: cellular signaling pathways, epigenetics, happenstance and the hope for a cure. Virology 45, 328-339. doi: 10.1016/j.virol.2014.02.008

Munshi, S. U., Panda, H., Holla, P., Rewari, B. B., and Jameel, S. (2014). MicroRNA150 is a potential biomarker of HIV/AIDS disease progression and therapy. PLoS One 9:e95920. doi: 10.1371/journal.pone.0095920

Ouellet, D. L., Plante, I., Barat, C., Tremblay, M. J., and Provost, P. (2009). Emergence of a complex relationship between HIV-1 and the microRNA pathway. Methods Mol. Biol. 487, 415-433. doi: 10.1007/978-1-60327-547-7_20

Pfeffer, S., Zavolan, M., Grasser, F. A., Chien, M., Russo, J. J., Ju, J., et al. (2004). Identification of virus-encoded microRNAs. Science 304, 734-736. doi: 10.1126/ science. 1096781
Poliseno, L., Salmena, L., Riccardi, L., Fornari, A., Song, M. S., Hobbs, R. M., et al. (2010). Identification of the miR-106b 25 microRNA cluster as a protooncogenic PTEN-targeting intron that cooperates with its host gene MCM7 in transformation. Sci. Signal. 3:ra29. doi: 10.1126/scisignal.2000594

Quick-Cleveland, J., Jacob, J. P., Weitz, S. H., Shoffner, G., Senturia, R., and Guo, F. (2014). The DGCR8 RNA-binding heme domain recognizes primary microRNAs by clamping the hairpin. Cell Rep. 7, 1994-2005. doi: 10.1016/j. celrep.2014.05.013

Ramalingam, P., Palanichamy, J. K., Singh, A., Das, P., Bhagat, M., Kassab, M. A., et al. (2014). Biogenesis of intronic miRNAs located in clusters by independent transcription and alternative splicing. RNA 20, 76-87. doi: 10.1261/rna.041814. 113

Reynoso, R., Laufer, N., Hackl, M., Skalicky, S., Monteforte, R., Turk, G., et al. (2014). MicroRNAs differentially present in the plasma of HIV elite controllers reduce HIV infection in vitro. Sci. Rep. 4:5915. doi: 10.1038/srep05915

Rodriguez, A., Griffiths-Jones, S., Ashurst, J. L., and Bradley, A. (2004). Identification of mammalian microRNA host genes and transcription units. Genome Res. 14, 1902-1910. doi: 10.1101/gr.2722704

Rosca, A., Anton, G., Botezatu, A., Temereanca, A., Ene, L., Achim, C., et al. (2016). miR-29a associates with viro-immunological markers of HIV infection in treatment experienced patients. J. Med. Virol. 88, 2132-2137. doi: 10.1002/ jmv. 24586

Seddiki, N., Phetsouphanh, C., Swaminathan, S., Xu, Y., Rao, S., Li, J., et al. (2013). The microRNA-9/B-lymphocyte-induced maturation protein-1/IL-2 axis is differentially regulated in progressive HIV infection. Eur. J. Immunol. 43, 510-520. doi: 10.1002/eji.201242695

Shen, C. J., Jia, Y. H., Tian, R. R., Ding, M., Zhang, C., and Wang, J. H. (2012). Translation of Pur-alpha is targeted by cellular miRNAs to modulate the differentiation-dependent susceptibility of monocytes to HIV-1 infection. FASEB J. 26, 4755-4764. doi: 10.1096/fj.12-209023

Squillace, N., Bresciani, E., Torsello, A., Bandera, A., Sabbatini, F., Giovannetti, C., et al. (2014). Changes in subcutaneous adipose tissue microRNA expression in HIV-infected patients. J. Antimicrob. Chemother. 69, 3067-3075. doi: 10.1093/ jac/dku264

Sung, T. L., and Rice, A. P. (2009). miR-198 inhibits HIV-1 gene expression and replication in monocytes and its mechanism of action appears to involve repression of cyclin T1. PLoS Pathog. 5:e1000263. doi: 10.1371/journal.ppat. 1000263

Swaminathan, S., Murray, D. D., and Kelleher, A. D. (2012a). The role of microRNAs in HIV-1 pathogenesis and therapy. AIDS 26, 1325-1334. doi: 10.1097/QAD.0b013e328352adca

Swaminathan, S., Suzuki, K., Seddiki, N., Kaplan, W., Cowley, M. J., Hood, C. L., et al. (2012b). Differential regulation of the Let-7 family of microRNAs in CD4+ T cells alters IL-10 expression. J. Immunol. 188, 6238-6246. doi: 10.4049/ jimmunol.1101196

Thapa, D. R., Hussain, S. K., Tran, W. C., D’Souza, G., Bream, J. H., Achenback, C. J., et al. (2014). Serum microRNAs in HIV-infected individuals as prediagnosis biomarkers for AIDS-NHL. J. Acquir. Immune Defic. Syndr. 66, 229-237. doi: 10.1097/QAI.0000000000000146

Triboulet, R., Mari, B., Lin, Y. L., Chable-Bessia, C., Bennasser, Y., Lebrigand, K., et al. (2007). Suppression of microRNA-silencing pathway by HIV-1 during virus replication. Science 315, 1579-1582. doi: 10.1126/science.1136319

Umbach, J. L., Yen, H. L., Poon, L. L., and Cullen, B. R. (2010). Influenza A virus expresses high levels of an unusual class of small viral leader RNAs in infected cells. MBio 1:e0204-10. doi: 10.1128/mBio.00204-10

Walker, B. D. (2007). Elite control of HIV Infection: implications for vaccines and treatment. Top. HIV Med. 15, 134-136.

Wang, F. S., Zhang, L., Douek, D., McMichael, A., Xu, X. N., and Lewin, S. R. (2018). Strategies for an HIV cure: progress and challenges. Nat. Immunol. 19, 1155-1158. doi: 10.1038/s41590-018-0242-8

Wang, P., Qu, X., Zhou, X., Shen, Y., Ji, H., Fu, Z., et al. (2015). Two cellular microRNAs, miR-196b and miR-1290, contribute to HIV-1 latency. Virology 486, 228-238. doi: 10.1016/j.virol.2015.09.016

Weiss, R. A. (1993). How does HIV cause AIDS? Science 260, 1273-1279.

Westerhout, E. M., Ooms, M., Vink, M., Das, A. T., and Berkhout, B. (2005). HIV1 can escape from RNA interference by evolving an alternative structure in its RNA genome. Nucleic Acids Res. 33, 796-804. doi: 10.1093/nar/gki220 
Whisnant, A. W., Bogerd, H. P., Flores, O., Ho, P., Powers, J. G., Sharova, N., et al. (2013). In-depth analysis of the interaction of HIV-1 with cellular microRNA biogenesis and effector mechanisms. mBio 4:e000193. doi: 10.1128/ mBio.00193-13

Witwer, K. W., Watson, A. K., Blankson, J. N., and Clements, J. E. (2012) Relationships of PBMC microRNA expression, plasma viral load, and CD4+ T-cell count in HIV-1-infected elite suppressors and viremic patients. Retrovirology 9:5. doi: 10.1186/1742-4690-9-5

Wu, Q., Song, R., Ortogero, N., Zheng, H., Evanoff, R., Small, C. L., et al. (2012). The RNase III enzyme DROSHA is essential for microRNA production and spermatogenesis. J. Biol. Chem. 287, 25173-25190. doi: 10.1074/jbc.M112. 362053

Wu, X., Zhang, L. L., Yin, L. B., Fu, Y. J., Jiang, Y. J., Ding, H. B., et al. (2017). Deregulated MicroRNA-21 expression in monocytes from HIV-infected patients contributes to elevated IP-10 secretion in HIV infection. Front. Immunol. 8:1122. doi: 10.3389/fimmu.2017.01122

Yang, J. S., and Lai, E. C. (2011). Alternative miRNA biogenesis pathways and the interpretation of core miRNA pathway mutants. Mol. Cell. 43, 892-903. doi: 10.1016/j.molcel.2011.07.024

Zhang, H. S., Wu, T. C., Sang, W. W., and Ruan, Z. (2012). MiR-217 is involved in Tat-induced HIV-1 long terminal repeat (LTR) transactivation by downregulation of SIRT1. Biochim. Biophys. Acta 1823, 1017-1023. doi: 10.1016/j. bbamcr.2012.02.014
Zhang, Y., Fan, M., Geng, G., Liu, B., Huang, Z., Luo, H., et al. (2014). A novel HIV-1-encoded microRNA enhances its viral replication by targeting the TATA box region. Retrovirology 11:23. doi: 10.1186/1742-4690-11-23

Zhang, Y., Yin, Y., Zhang, S., Luo, H., and Zhang, H. (2016). HIV-1 infectioninduced suppression of the Let-7i/IL-2 axis contributes to CD4(+) T cell death. Sci. Rep. 6:25341. doi: 10.1038/srep25341

Zinovyeva, A. Y., Veksler-Lublinsky, I., Vashisht, A. A., Wohlschlegel, J. A., and Ambros, V. R. (2015). Caenorhabditis elegans ALG-1 antimorphic mutations uncover functions for argonaute in microRNA guide strand selection and passenger strand disposal. Proc. Natl. Acad. Sci. U.S.A. 112, E5271-E5280. doi: $10.1073 /$ pnas. 1506576112

Conflict of Interest Statement: The authors declare that the research was conducted in the absence of any commercial or financial relationships that could be construed as a potential conflict of interest.

Copyright (C) $2018 \mathrm{Su}, \mathrm{Fu}, \mathrm{Liu}, \mathrm{Wu}, \mathrm{Ma}$, Zeng, Zhang, Lian and Wu. This is an open-access article distributed under the terms of the Creative Commons Attribution License (CC BY). The use, distribution or reproduction in other forums is permitted, provided the original author(s) and the copyright owner(s) are credited and that the original publication in this journal is cited, in accordance with accepted academic practice. No use, distribution or reproduction is permitted which does not comply with these terms. 